\title{
BMJ Open Acceptability and feasibility of dual HIV and syphilis point-of-care testing for early detection of infection among pregnant women in China: a prospective study
}

\author{
Qian Wang, ${ }^{1}$ Po-Lin Chan, ${ }^{2}$ Lori M Newman, ${ }^{3}$ Li-Xia Dou, ${ }^{1}$ Xiao-Yan Wang, ${ }^{1}$ \\ Ya-Ping Qiao, ${ }^{1}$ Min Su, ${ }^{1}$ Xiang-Sheng Chen, ${ }^{4}$ Xi Jin, ${ }^{1}$ Ai-Ling Wang ${ }^{1}$
}

To cite: Wang Q, Chan P-L, Newman LM, et al. Acceptability and feasibility of dual HIV and syphilis point-of-care testing for early detection of infection among pregnant women in China: a prospective study. BMJ Open 2018;8:e020717. doi:10.1136/ bmjopen-2017-020717

- Prepublication history and additional material for this paper are available online. To view please visit the journal (http:// dx.doi.org/10.1136/bmjopen2017-020717).

Received 21 November 2017 Revised 28 July 2018 Accepted 8 August 2018

Check for updates

(C) Author(s) (or their employer(s)) 2018. Re-use permitted under CC BY-NC. No commercial re-use. See rights and permissions. Published by BMJ.

For numbered affiliations see end of article.

Correspondence to

Ai-Ling Wang;

ailing@chinawch.org.cn

\section{ABSTRACT}

Objective To assess the feasibility and acceptability of using WHO prequalified combined dual HIV/syphilis rapid diagnostic tests (RDT) for same-day results in antenatal care (ANC) clinics.

Methods This is a pragmatic implementation study using quantitative approach to evaluate outcomes. Antenatal clinic attendees from 21 rural and urban township hospitals in two provinces of China were offered with free dual RDTs testing that included HIV and syphilis, in addition to the routine blood tests. Study outcomes included testing uptake before and during dual RDT use, test feasibility and acceptability among pregnant women. Regression model was used to assess acceptance of RDT testing.

Results In total, 1787 out of 1828 pregnant women attending ANC received the RDT testing. Testing uptake among pregnant women in their first and second trimester increased from $76.0 \%(2438 / 3269)$ using standard blood testing to $90.1 \%(1626 / 1787)$ with concurrent RDT use $\left(\chi^{2}=197.1, p<0.001\right)$. Among 1787 pregnant women who received RDT tests, 98.3\% (1757/1787) participants were given test result the same day. Positive proportions of HIV and syphilis screened with RDT were $0.06 \%(1 / 1787)$ and $1.0 \%(18 / 1787)$, respectively. Regression analysis indicated that women who did not receive syphilis or HIV testing before were less likely to accept dual RDT (OR $0.28,95 \% \mathrm{Cl} 0.10$ to 0.75 ). Acceptance for dual RDT testing at second or third antenatal visit was lower compared with the first visit $(\mathrm{OR} 0.37,95 \% \mathrm{Cl} 0.15$ to 0.94).

Conclusion Combined dual HIV/syphilis RDT with sameday results increased uptake of HIV and syphilis testing among pregnant women at primary healthcare facilities. Given the diversity of testing capacities among health services especially in rural areas in China, the dual RDT kit is feasible tool to improve testing uptake among pregnant women.

\section{INTRODUCTION}

Eliminating HIV and syphilis as public health threats by 2030 is an ambitious global health goal articulated by WHO to support the health
Strengths and limitations of this study

- The study included a very large sample of women attending antenatal clinics at primary care level, the representative of services for women in China.

- With the focus on real-world implementation, we used quantitative methods to understand the feasibility and outcomes of using a new technology (dual rapid diagnostic test) to test HIV and syphilis. The application lends strength to the study and interpretation of results.

- The limitation includes that clinics were purposively selected, based on the willingness of hospital administration to participate in the study. This may have introduce some degree of positive bias.

targets of the 2030 Agenda for Sustainable Development Goals. ${ }^{1}$ The syndemics of HIV and syphilis among pregnant women is one of the greatest challenges in public health and maternal health globally. The burden among pregnant women is substantial. In 2013, there were more than 1.4 million pregnant women living with HIV in low-income and middle-income countries, with an estimated 240000 children newly infected with HIV. ${ }^{2}$ In 2012, an estimated 930000 maternal syphilis infections caused 350000 adverse pregnancy outcomes including 143000 early fetal deaths and stillbirths, 62000 neonatal deaths, 44000 preterm or low weight births and 102000 infected infants worldwide. ${ }^{3}$

In the last decade, notable improvements have been achieved in advancing services for HIV and sexually transmitted infections. The increase in numbers of pregnant women screened for HIV and syphilis, together with increased access to treatment help underline the feasibility of dual elimination of motherto-child transmission (EMTCT) of HIV and 
syphilis. ${ }^{1}$ In China, testing and counselling are the primary intervention steps in the package of essential services for prevention of MTCT (PMTCT). PMTCT interventions include provision of free antiretroviral (ARV) treatment and diagnosis for exposed infants. ${ }^{4}$

WHO and The Joint United Nations Programme on HIV/AIDS recommend a dual strategy for EMTCT of HIV and syphilis. ${ }^{2}$ Global and regional initiatives for dual EMTCT of HIV and syphilis were launched in 2011. China initiated the free integrated PMTCT (iPMTCT) of HIV, syphilis and HBV programme in 2011 which included universal coverage of HIV, syphilis and hepatitis B antenatal testing. Early detection and timely intervention are critical components. As public health interventions, antenatal syphilis screening and treatment are proved to be cost saving in moderate and high-prevalence settings and cost-effective even when prevalence is less than $1 \% .^{5-7} \mathrm{In}$ China, HIV MTCT decreased from an estimated $34.8 \%$ in 2004 to $6.1 \%$ in 2014 after expansion of universal access to HIV antenatal screening for all pregnant women and free ARV prophylaxis for HIV-infected pregnant women with replacement feeding for HIV-exposed infants. ${ }^{8}$ Reported congenital syphilis increased from 25.5 per 100000 live births in 2005 to 60.8 per 100000 live births in 2009. The situation possibly related to increasing access to syphilis diagnosis. ${ }^{4}$ Apparently, to achieve the goal of dual EMTCT of HIV and syphilis, early diagnosis and timely intervention are key steps in the cascade of interventions then to reduce new infections among infants.

Worldwide, access to quality-assured diagnosis and treatment services is restricted or absent in many resource-limited settings across low-income and middle-income countries, like Mongolia, Ghana and China. ${ }^{5}{ }^{10}$ In order to improve the number of women tested and treated, innovative strategies are imperative. A number of single rapid diagnostic tests (RDTs) for HIV or syphilis have been developed and scaled up across the world. ${ }^{811} \mathrm{~A}$ combination of these two rapid point-of-care diagnostic test kit enables syphilis and HIV testing to be offered concurrently at any antenatal care (ANC) setting. These dual RDTs have shown great prospect based on sensitivity and specificity assessments conducted in laboratory evaluation, and are low cost and commercially available. ${ }^{12-16}$ Dual RDTs can concurrently test antibodies to HIV and Treponema pallidum antigens, also ensuring the test to be conducted in a single visit with provision of results within the same day. This approach offers opportunities to improve testing uptake among pregnant women and thus achieve the aim of EMTCT of HIV and syphilis. ${ }^{16} 17$

The current universal antenatal testing strategy in the national PMTCT programme in China for HIV and syphilis is based mainly on the use of the HIV enzyme immunoassay and rapid plasma reagin non-treponemal test for syphilis. These tests require venous blood samples and may take several days until the results return. In addition, the tests are technically demanding and require laboratory equipment, which is not widely available in many resource-limited settings, especially in rural or hard-to-reach regions in the country. These conditions impede equitable access of HIV and syphilis tests to all pregnant women in China. The application of dual HIV and syphilis RDTs could be helpful to relieve these problems.

This study aims to evaluate the feasibility and acceptability of dual HIV and syphilis rapid diagnostics for early testing among pregnant women in comparison to routine assay tests in primary care facilities in China.

\section{METHODS}

\section{Study design}

This pragmatic implementation study used quantitative approach to evaluate outcomes. Quantitative data were collected from pregnant women attending ANC clinics.

\section{Study setting, participants and public involvement}

Pregnant women attending the Yuantan township hospital (Guangdong Province) and the Funan township hospital (Anhui province) in China from February to July in 2015 were invited to enrol. Baseline data were collected from the past 3 months from the ANC registry of these two sites from October 2014 to January 2015.

The following inclusion and exclusion criteria were used to recruit pregnant women for the feasibility study. (1) Inclusion criteria: women attending the antenatal clinic at the study sites and unaware of their HIV and syphilis status when they were enrolled into the study. (2) Exclusion criteria: women less than 16 years of age, unable to provide informed consent, had already been tested and aware of their HIV and/or syphilis status or with prior participation of the evaluation study.

Patients were not involved in the design, conduct or analysis of the study. The preliminary results summary was collated and presented on propaganda posters within two township hospitals, and also passed by the ANC lectures in prenatal health education programmes.

\section{Variables and data resources}

The number and percentage of women attending ANC, the number and percentage of women tested for HIV using the routine tests (ELISA; chemiluminescence immunoassay, CLIA; particle agglutination assay, PA) and the number and percentage of women tested for syphilis with both treponemal and non-treponemal antibodies testing were collected. All enrolled pregnant women provided informed consent before they were interviewed or tested with RDT.

At the time of introduction of the dual RDTs, all ANC attendees were provided HIV, syphilis testing and counselling, which included RDT-related information as well as information on the routine HIV or syphilis testing method in the participating sites. At the same time, all ANC attendees were surveyed to collect basic information on sociodemographic characteristics, partner testing, self-reporting previous history of pregnancy, HIV and 
syphilis testing and treatment, and current HIV and syphilis testing and treatment management.

\section{Sample size}

To estimate a single proportion with an adequate level of precision, we assumed a 95\% CI for the proportion and assumed that the unknown proportion to be 0.50 with a precision of no wider than 0.05 (ie, $\mathrm{m} \leq 0.025$ ). With the formula: $n \geq(2 / m) 2 \times p \times(1 p)$, we estimated that a total of at least 1600 pregnant women were needed for study.

\section{Dual HIV and syphilis tests}

All consenting ANC attendees were offered with dual HIV/syphilis RDT kits. The dual tests were conducted with finger stick sample, unless the venous blood was collected for other obstetric blood testing. A total of 1633 out of $1787(91.1 \%)$ pregnant women provided fingerprick blood samples, and the other $154(0.09 \%)$ provided only venous blood samples. Test results were recorded in the questionnaires (online supplementary material). Reference tests such as ELISA, CLIA or PA for RDT results comparison were conducted at the ANC laboratory in the clinic or hospital. Positive results using RDT tests were revaluated by routine national protocols, which required confirmation by the National Reference Laboratory of National Center for AIDS Prevention and Control. All attendees were notified of results and offered treatment if the confirmed test results were positive.

\section{Statistical analysis}

Data collected via the questionnaires (online supplementary material) were entered in Epidata V.3.0 and were checked by staff. We used SAS V.19.0 to measure the outcomes and compare the outcome indicators (willingness for RDT, early testing coverage) at the baseline and after introduction of the dual RDTs. $\chi^{2}$ tests were used to examine the change in early testing coverage before and after implementing RDT among pregnant women.

Acceptability was defined as a pregnant woman actually receiving dual RDT testing. Acceptability of dual HIV/ syphilis RDTs by ANC attendees was independently tested against the following factors: age, sociodemographic characteristics, history of pregnancy, HIV and syphilis testing and treatment, willingness to test and distance to ANC. A multivariate logistic model was used to determine predictors of acceptability of RDT among pregnant women while adjusting for confounding factors. Variables with $\mathrm{p}<0.10$ in the univariate analyses were eligible for inclusion in the multivariate model. Variables were retained in the final model only if they were statistically significantly associated $(\mathrm{p}<0.05)$ with the outcome or deemed to be of a priori importance. A backward approach was used to select variables for the final model. The adjusted ORs with $95 \%$ CI were reported. P values were used to measure strength of association and identify statistically significant results. A $p<0.05$ was considered as a statistically significant association.

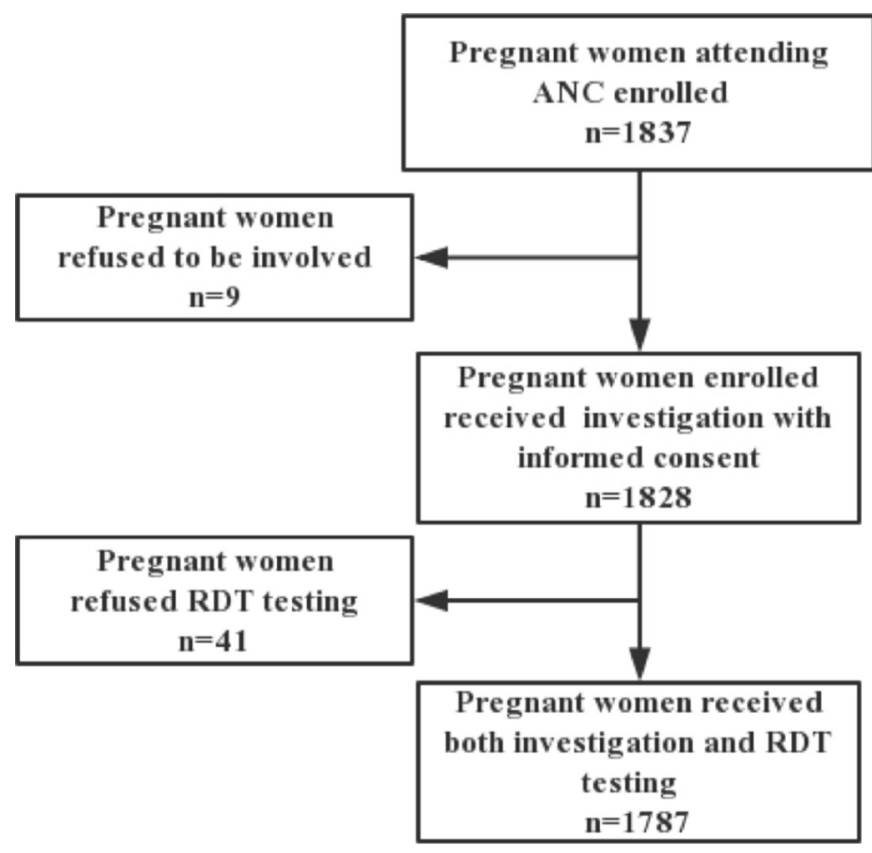

Figure 1 Study of acceptability and feasibility of dual HIV and syphilis point-of-care testing for early detection of infection among pregnant women in China sample enrolment flow chart. ANC, antenatal care; RDT, rapid diagnostic test.

\section{RESULTS}

\section{Demographic characteristics of pregnant women}

Between February and July 2015, a total of 1828 pregnant women eligible for the study were offered with RDT tests and also completed questionnaires (figure 1). Same like RDTs, 6 of them were found infected with HIV and 17 were tested positive for syphilis infection with reference tests. The median age of the cohort was 23 years (range 18-47 years). Local residents accounted for $96.9 \%$ $(1772 / 1828)$ (table 1$)$. The majority of the participants $(88.0 \%(1607 / 1828))$ had education of senior middle school or above and 6.5\% (119/1828) received junior middle school education or below. More than half of participants $(65.5 \%(1196 / 1828))$ were farmers, and $33.4 \%(606 / 1828)$ of pregnant women were in their first pregnancy and $56.0 \%(1010 / 1811)$ had already gave birth to at least one child. The average gestational age of pregnancy at enrolment was 20.4 weeks (range 8-43 weeks).

\section{Accessibility to ANC clinics}

Participants took a median time of 34.5 min (range 5 min to 4 hours) to travel to ANC clinics. The majority of pregnant women $(60.0 \%(1083 / 1817))$ took motor or autobikes, $32.7 \%$ (589/1817) travelled by bus to clinics and $7.3 \%(137 / 1871)$ walked to ANC clinics.

\section{Uptake of dual HIV and syphilis rapid testing}

In total, testing uptake among pregnant women in their first and second trimester increased from $76.0 \%$ (2438/3269) using standard blood testing to $90.1 \%$ $(1626 / 1787)$ with concurrent HIV and syphilis RDT test kit $\left(\chi^{2}=197.1, \mathrm{p}<0.001\right)$. 
Open access

Table 1 Univariate analysis for acceptability of RDT for early testing among pregnant women in China

\section{Accepted}

Indicators

All

n $\%$

\section{Unaccepted}

Hukou

\begin{tabular}{|c|c|c|c|c|c|c|c|}
\hline Local resident & 1771 & 1718 & 97.0 & 53 & 3.0 & & \\
\hline Temporary resident & 27 & 24 & 92.3 & 3 & 7.7 & & \\
\hline Migration people & 19 & 18 & 94.7 & 1 & 5.3 & & \\
\hline Age & & & & & & & $0.81^{*}$ \\
\hline$<25$ & 1029 & 994 & 96.9 & 35 & 3.4 & & \\
\hline $30-34$ & 139 & 134 & 96.4 & 5 & 3.6 & & \\
\hline $35-39$ & 42 & 41 & 97.6 & 1 & 2.4 & & \\
\hline$>40$ & 10 & 10 & 100 & 0 & 0 & & \\
\hline Education & & & & & & & $0.88^{*}$ \\
\hline Primary & 119 & 117 & 98.3 & 2 & 1.7 & & \\
\hline Other & 2 & 2 & 100 & 0 & 0 & & \\
\hline Occupation & & & & & & & $0.24^{*}$ \\
\hline Farmer (peasant-worker) & 1585 & 1536 & 96.9 & 49 & 3.1 & & \\
\hline Unemployed & 156 & 153 & 98.1 & 3 & 1.9 & & \\
\hline Other & 86 & 81 & 94.2 & 5 & 5.8 & & \\
\hline First ANC visit & & & & & & & $0.02^{*}$ \\
\hline Yes & 898 & 880 & 98.0 & 18 & 2.0 & & \\
\hline No & 922 & 887 & 96.2 & 35 & 3.8 & & \\
\hline No & 790 & 763 & 96.6 & 27 & 3.4 & & \\
\hline Other & 15 & 14 & 93.3 & 1 & 6.7 & & \\
\hline History of abortion & & & & & & 4.5 & 0.10 \\
\hline Yes & 291 & 287 & 98.6 & 4 & 1.4 & & \\
\hline No & 1507 & 1456 & 96.6 & 51 & 3.4 & & \\
\hline Other & 30 & 28 & 93.3 & 2 & 6.7 & & \\
\hline Having children & & & & & & 12.7 & $<0.001$ \\
\hline Yes & 1010 & 983 & 97.3 & 27 & 2.7 & & \\
\hline No & 801 & 774 & 96.6 & 27 & 3.4 & & \\
\hline Other & 14 & 14 & 82.4 & 3 & 17.6 & & \\
\hline gestational weeks & & & & & & 30.2 & $<0.001$ \\
\hline$\sim 12$ & 578 & 561 & 97.1 & 17 & 2.9 & & \\
\hline $13-27$ & 669 & 656 & 98.1 & 13 & 1.9 & & \\
\hline
\end{tabular}

Continued 
Table 1 Continued

\begin{tabular}{|c|c|c|c|c|c|c|c|}
\hline \multirow[b]{2}{*}{ Indicators } & \multirow[b]{2}{*}{ All } & \multicolumn{2}{|c|}{ Accepted } & \multicolumn{2}{|c|}{ Unaccepted } & \multirow[b]{2}{*}{$\chi^{2}$} & \multirow[b]{2}{*}{$P$ values } \\
\hline & & $\mathbf{n}$ & $\%$ & $\mathbf{n}$ & $\%$ & & \\
\hline $28 \sim$ & 565 & 542 & 95.9 & 23 & 4.1 & & \\
\hline Other & 16 & 12 & 75.0 & 4 & 25.0 & & \\
\hline Preference of delivery place & & & & & & & $0.11^{*}$ \\
\hline Township level & 976 & 937 & 96.0 & 39 & 4.0 & & \\
\hline County level & 703 & 690 & 98.2 & 13 & 1.8 & & \\
\hline City level & 64 & 62 & 96.9 & 2 & 3.1 & & \\
\hline Private hospitals & 11 & 11 & 100 & 0 & 0 & & \\
\hline No idea & 74 & 71 & 96.0 & 3 & 4.0 & & \\
\hline Time from home to ANC & & & & & & & $0.09^{*}$ \\
\hline$\sim 30 \mathrm{~min}$ & 1323 & 1280 & 96.9 & 43 & 3.1 & & \\
\hline$\sim 60 \mathrm{~min}$ & 350 & 343 & 98.0 & 7 & 2.0 & & \\
\hline$\sim 120 \mathrm{~min}$ & 141 & 136 & 96.5 & 5 & 3.5 & & \\
\hline 120 min and above & 14 & 12 & 85.7 & 2 & 12.3 & & \\
\hline HIV testing before & & & & & & 2.00 & 0.37 \\
\hline Yes & 717 & 700 & 97.7 & 17 & 2.3 & & \\
\hline No & 190 & 182 & 95.8 & 8 & 4.2 & & \\
\hline Other & 213 & 206 & 96.7 & 7 & 3.3 & & \\
\hline Syphilis testing before & & & & & & 2.9 & 0.23 \\
\hline Yes & 695 & 680 & 97.8 & 15 & 2.2 & & \\
\hline No & 184 & 177 & 96.2 & 7 & 3.8 & & \\
\hline Other & 225 & 216 & 96.0 & 9 & 4.0 & & \\
\hline
\end{tabular}

${ }^{*}$ Fisher accurate $\chi^{2}$.

ANC, antenatal care; RDT, rapid diagnostic test.

In 1828 pregnant women who were enrolled in the study, 1787 underwent RDT testing. Positive RDT screening results for HIV and syphilis were $0.06 \%(1 / 1787)$ and $1.0 \%$ (18/1787), respectively. RDT results were $100 \%$ correspond to national confirmatory tests.

Among those who had previously been pregnant, $64.3 \%(699 / 1087)$ and $63.1 \%(677 / 1037)$ of them self-reported having received HIV and syphilis tests during their first pregnancy. Among these women previously been tested, two were positive for HIV and six for syphilis.

\section{Willingness to accept HIV/syphilis RDT dual testing}

Among 1828 participants who were offered with RDT tests, $1794(95.3 \%)$ expressed willing to undergo rapid tests at once and $34(4.7 \%)$ were reluctant. Before the RDT administered, participants received pretest education and filled in questionnaires (online supplementary material). These participants still tested via routine screening tests for HIV and syphilis. In total, 1771 pregnant women received RDT testing and 23 refused rapid testing. Of the 23 refusals, 13 stated that they had been tested previously and 10 wanted their blood samples to be taken during the third trimester rather than the first.

\section{Associated factors with RDT testing acceptability}

Regression analysis indicated that demographic characteristics, pregnancy history, gestational week, distance to ANC or preference of delivery place did not affect the acceptability of RDT test among participating pregnant women (table 1). Acceptability of RDT testing was statistically lower in women who had not received HIV or syphilis testing before compared with those who had previously tested (OR 0.28 ; $95 \%$ CI 0.10 to 0.75 ). Acceptance of dual RDT testing at the second or third antenatal visits was lower compared with those were at their first visit (OR $0.37,95 \%$ CI 0.15 to 0.94 ) (tables 1 and 2).

\section{Feasibility of dual HIV and syphilis rapid testing for early} testing among pregnant women

Among 1787 pregnant women who received RDT testing, $98.3 \%(1757 / 1787)$ of participants were given back their test results on the same day. Among those who did not received RDT testing result on the same day, $65.6 \%(20 / 30)$ did not return to ANC for test results. Six (18.8\%) pregnant women left ANC before RDT testing results were provided, and $14.1 \%(4 / 30)$ thought the test results would be provided later. Among 1757 pregnant women received RDT testing results, 54.9\% (965/1757) 
Table 2 Regression analysis and factors associated with rapid diagnostic test acceptability $(n=1828)$

\begin{tabular}{|c|c|c|c|c|c|c|c|}
\hline \multirow[b]{2}{*}{ Indicator } & \multirow[b]{2}{*}{ Intercept } & \multirow[b]{2}{*}{ SE } & \multirow[b]{2}{*}{ Wald } & \multirow[b]{2}{*}{$P$ values } & \multirow[b]{2}{*}{ OR } & \multicolumn{2}{|c|}{$95 \%$ OR } \\
\hline & & & & & & Lower & Upper \\
\hline Intercept & 3.31 & 1.78 & 3.47 & 0.06 & & & \\
\hline $\begin{array}{l}\text { Syphilis testing before } \\
\text { no/yes }\end{array}$ & -1.29 & 0.51 & 6.33 & 0.01 & 0.28 & 0.10 & 0.75 \\
\hline
\end{tabular}

receiving their RDT results within $30 \mathrm{~min}, 20.7 \%$ (364/1757) received results within 30-60 min and 428 $(24.4 \%)$ received results longer than 1 hour later.

\section{DISCUSSION}

This study showed good feasibility of use of dual RDTs for early HIV and syphilis testing among pregnant women. We noted marked improvements in uptake of HIV and syphilis testing with use of RDTs in China. This is an important finding because in some countries, despite the majority of pregnant women having access to ANC, only a small proportion undergo HIV and/or syphilis screening. ${ }^{10} 12$ In 2012, more than $60 \%$ of pregnant women in Nigeria received ANC, but only $33 \%$ were screened for syphilis, and in Northeast areas of the country only less than $10 \%$ of pregnant women were screened. ${ }^{2}$ China's iPMTCT programme reached 13.1 million pregnant women in 2013. Pregnant women HIV antibodies testing showed an increase in testing rates from $85.4 \%$ in 2009 to $97.3 \%$ in 2013. However, HIV, syphilis and HBV testing coverage in pregnancy varies tremendously in different regions of China. ${ }^{12}$ In Sichuan, Yunnan and Xinjiang provinces, HIV, syphilis and HBV testing during pregnancy was $79.4 \%, 68.4 \%$ and $69.9 \%$, respectively. ${ }^{12}$ In some counties in Guangdong province where the overall prevalence of $\mathrm{HBV}$ is high, the three-disease testing rates during pregnancy was found to be low. ${ }^{14}$

Several studies in China have reported that lack of HIV or syphilis testing during pregnancy and labour, and inadequately equipped laboratories with trained personnel. These are major barriers to testing uptake. ${ }^{1318}$ Therefore, new approaches to HIV and syphilis screening should be adopted and promoted. Point-of-care tests can accelerate access to testing and subsequent connection to treatment and care services by shortening the time to result in a group of women and their families who are in close contact with health services. ${ }^{17}$

The standard test methods require traditional assays and well-equipped laboratories with trained personnel, which impedes testing uptake and interventions. Timely diagnosis for HIV-infected pregnant women enables provision of ARVs to reduce risk of transmission to their infants, and the effectiveness of these interventions has been demonstrated in China. ${ }^{20}$ Our study indicated testing uptake among pregnant women in their first and second trimester increased by $14 \%$ compared with baseline survey. This dual test is particularly timely given WHO and UNAIDS recommendations for a dual strategy for the prevention of HIV and syphilis as well as WHO policy on sexually transmitted infections testing for key populations. 11821

It is worth to be noticed that the acceptance of dual RDT testing at the second or third antenatal visit was lower compared with that of the first visit. This is consistent with previous findings. ${ }^{10}$ Punguyire et al found pregnant women HIV testing uptake differed according to maternal healthcare. ${ }^{10}$ Similar findings have been reported by other studies in China. ${ }^{12-1420}$ Receiving HIV or syphilis testing previously also affected RDT acceptance among pregnant women. This is probably because knowledge of one's status would decrease their requirement to have another test.

The limitations of this survey include that the study was conducted in purposively selected township clinics, according to the willingness of hospital administration to participate in the study. This may introduce some degree of positive bias. However, the objective was to assess realworld pragmatic field scenarios and as the study included a large number of participating sites and pregnant women, we opined that the results faithfully reflect the actual experience of providers and women.

In summary, our study showed that dual RDTs with same-day results are acceptable by pregnant women and feasible to promote in China. Dual RDTs use at decentralised healthcare facilities were feasible. It simplifies the access to diagnosis, the critical first step to the cascade of PMTCT care for those who are infected. ${ }^{22} 23$ Moreover, in order to achieve the goal of dual EMTCT, reaching high coverage of testing among all pregnant women is essential. ${ }^{2425}$ Thus, RDTs would also be useful approaches to expand access to combined HIV/syphilis diagnosis among hard to reach populations and geographical areas in China as well as other countries. The next step planned for the scale up of RDT testing in the national programme is to promote the use of quality-assured diagnostics across the country, particularly in decentralised health facilities. Some township hospitals of pilot areas without well-equipped laboratories have already begun 
procuring dual or triple rapid testing kits for routine use. Moreover, widespread introduction of RDT tests could accelerate the scale up of screening for HIV and syphilis among pregnant women throughout China.

\section{Author affiliations}

${ }^{1}$ National Center for Women and Children's Health, Chinese Center for Disease Control and Prevention, Beijing, China

${ }^{2}$ Division of Communicable Diseases, World Health Orgnization China Office, Beijing, China

${ }^{3}$ Department of Reproductive Health Research, World Health Organization, Geneva, Swaziland

${ }^{4}$ National Center for Sexually Transmitted Disease Control, Chinese Academy of Medical Science and Peking Union Medical College Institute of Dermatology, Nanjing, China

Acknowledgements This study was funded by the UNDP-UNFPA-WHO- World Bank Special Programme of Research, Development and Research training in Human Reproduction in WHO.

Contributors QW, LMN, X-SC and A-LW conceived and designed the study, reviewed and revised manuscript. QW and P-LC performed the study, supervised data collection, analysed the data, drafted the initial manuscript and revised the manuscript. L-XD, X-YW, Y-PQ, XJ and MS contributed to data collection and analysis, and reviewed the manuscript. All authors approved the final manuscript as submitted and agree to be accountable for all aspects of the work.

Funding This study was funded by the UNDP-UNFPA-WHO-World Bank Special Programme of Research, Development and Research training in Human Reproduction in WHO.

Competing interests None declared.

Patient consent Obtained.

Ethics approval Ethical clearance was provided by the Institutional Review Board of the Center for National Women and Children's Health at the China Center for Disease Control. The study protocol was approved by the CenterCentre for National Women and Children's Health (IRB Number:FY-2014-001).

Provenance and peer review Not commissioned; externally peer reviewed.

Data sharing statement Original data are available and can be accessed by contacting A-LW. Review Board of Center for National Women and Children's Health within China Center for Disease Control conducted the participant deidentification. All participants permitted this research-oriented data usage of their blood sample.

Open access This is an open access article distributed in accordance with the Creative Commons Attribution Non Commercial (CC BY-NC 4.0) license, which permits others to distribute, remix, adapt, build upon this work non-commercially, and license their derivative works on different terms, provided the original work is properly cited, appropriate credit is given, any changes made indicated, and the use is non-commercial. See: http://creativecommons.org/licenses/by-nc/4.0/.

\section{REFERENCES}

1. WHO. Global health sector strategy on sexually transmitted infections: TOWARDS ENDING STIs, 2016-2021. http://www.who.int/ reproductivehealth/publications/rtis/ghss-stis/en/

2. The gap report 2014: children and pregnant women living with HIV/ AIDS. http://www.unaids.org/sites/default/files/media_asset/09 Chil drenandpregnantwomenlivingwithHIV.pdf

3. Wijesooriya NS, Rochat RW, Kamb ML, et al. Global burden of maternal and congenital syphilis in 2008 and 2012: a health systems modelling study. Lancet Glob Health 2016;4:e525-e533.

4. Gong XD, Yue XL, Teng F, et al. Syphilis in China from 2000 to 2013: epidemical trends and characteristics. Chin J Dermatol 2014;5:310-5.
5. WHO. Global guidance on criteria and processes for validation: elimination of mother-to-child transmission of HIV and syphilis. 2nd edn. Geneva: World Health Organization, 2017.

6. Owusu-Edusei K, Gift TL, Ballard RC. Cost-effectiveness of a dual non-treponemal/treponemal syphilis point-of-care test to prevent adverse pregnancy outcomes in sub-Saharan Africa. Sex Transm Dis 2011;38:997-1003.

7. Blandford JM, Gift TL, Vasaikar S, et al. Cost-effectiveness of on-site antenatal screening to prevent congenital syphilis in rural eastern Cape Province, Republic of South Africa. Sex Transm Dis 2007;34:S61-S66.

8. National Health and Family Planning Commission of the People's Republic of China. 2015 China AIDS response progress report, 2015. http://www.unaids.org/sites/default/files/country/documents/CHN narrative_report_2015.pdf

9. Munkhuu B, Liabsuetrakul T, Chongsuvivatwong V, et al. Coverage of antenatal syphilis screening and predictors for not being screened in Ulaanbaatar, Mongolia. Sex Transm Dis 2006;33:284-8.

10. Punguyire D, Mahama E, Letsa T, et al. Factors associated with syphilis screening uptake among pregnant women in health facilities in Brong Ahafo Region of Ghana. Matern Health Neonatol Perinatol 2015;1:7.

11. Watson-Jones $D$, Oliff M, Terris-Prestholt $F$, et al. Antenatal syphilis screening in sub-Saharan Africa: lessons learned from Tanzania. Trop Med Int Health 2005;10:934-43.

12. Wang XY, Fang LW, Wang F, et al. Analysis of HIV, syphilis and HBV testing services among pregnant women in some counties of China. Chinese Journal of AIDS \& STD 2013;12:880-2.

13. Li S, Zhang S, Li Y, et al. [Impact factors related to HIV voluntary counseling and testing of pregnant women in Liangshan $\mathrm{Yi}$ Autonomous Prefecture, Sichuan]. Zhonghua Yu Fang Yi Xue Za Zhi 2015;49:1014-6.

14. Tang LY, Zhao QG, Li B, et al. Analysis of HIV, syphilis and HBV testing services among pregnant women in Guangdong. Chinese Journal of AIDS \& STD 2015;9:762-5.

15. Omoding D, Katawera V, Siedner M, et al. Evaluation of the SD Bioline HIV/Syphilis Duo assay at a rural health center in Southwestern Uganda. BMC Res Notes 2014;7:746.

16. Gliddon HD, Peeling RW, Kamb ML, et al. A systematic review and meta-analysis of studies evaluating the performance and operational characteristics of dual point-of-care tests for HIV and syphilis. Sex Transm Infect 2017;93(S4):S3-S15.

17. WHO. WHO information note on the use of dual HIV/Syphilis Rapid Diagnostic Tests (RDT), 2017. http://apps.who.int/iris/bitstream/ 10665/252849/1/WHO-RHR-17.01-eng.pdf

18. Wang AL, Qiao YP, Wang LH, et al. Integrated prevention of mother-to-child transmission for human immunodeficiency virus, syphilis and hepatitis B virus in China. Bull World Health Organ 2015;93:52-6.

19. Bristow CC, Adu-Sarkodie Y, Ondondo RO, et al. Multisite laboratory evaluation of a dual Human Immunodeficiency Virus (HIV)/syphilis point-of-care rapid test for simultaneous detection of HIV and syphilis infection. Open Forum Infect Dis 2014;1:ofu015.

20. Wang $Q$, Wang L, Fang L, et al. Timely antiretroviral prophylaxis during pregnancy effectively reduces HIV mother-to-child transmission in eight counties in China: a prospective study during 2004-2011. Sci Rep 2016;6:34526.

21. Yin YP, Ngige E, Anyaike C, et al. Laboratory evaluation of three dual rapid diagnostic tests for HIV and syphilis in China and Nigeria. Int $J$ Gynaecol Obstet 2015;130(Suppl 1):S22-S26.

22. Zhang D, Meng S, Xu P, et al. Experience of offering HIV rapid testing to at-risk patients in community health centers in eight Chinese cities. PLoS One 2014;9:e86609.

23. Sun $\mathrm{Y}$, Hewan-Lowe $\mathrm{K}, \mathrm{Wu} \mathrm{Q}$, et al. HIV screening and awareness survey for pregnant women in a remote area in Xinjiang uyghur autonomous region of China. Open AIDS J 2011;5:96-101.

24. Gu WM, Yang Y, Wang QZ, et al. Comparing the performance of traditional non-treponemal tests on syphilis and non-syphilis serum samples. Int J STD AIDS 2013;24:919-25.

25. Cheng JQ, Zhou H, Hong FC, et al. Syphilis screening and intervention in 500,000 pregnant women in Shenzhen, the People's Republic of China. Sex Transm Infect 2007;83:347-50. 